\title{
artigo
}

\section{Lombalgia em portadores de paraparesia espástica tropical/mielopatia associada ao HTLV 1}

\author{
Lombalgia in tropical spastic parapharry / myelopathy associated with HTLV 1 \\ Lombalgia en pacientes con paraaparesis / mielopatía tropical espástica asociada con HTLV 1
}

\begin{abstract}
RESUMO
A dor lombar relacionada à Paraparesia Espástica Tropical/Mielopatia associada ao HTLV 1 (PET/MAH) torna-se relevante por esta ser um sintoma clínico freqüente nesses pacientes. Objetivos foram avaliar a dor lombar e incapacidades. MÉTODOS: Foram avaliados 15 portadores de PET/MAH, através de três questionários: Escala Analógica Visual (EVA), Índice de Katz e Questionário Roland-Morris de Incapacidades para dor lombar. RESULTADOS: Houve prevalência de dor lombar (93,30\%), sendo que esta dor apresentou-se de forma localizada $(85,71 \%)$. A intensidade da dor era moderada $(66,7 \%)$, a maioria $(60,0 \%)$ era independente, entretanto $(53,3 \%)$ destes tem incapacidade funcional associada à dor lombar. CONCLUSÃO: Este estudo mostrou alta prevalência de dor lombar, quanto maior o nível de dor referido pelos pacientes, maior será o nível de limitação funcional apresentados pelos mesmos por haver relação direta entre ambos.
\end{abstract}

DESCRITORES: Dor lombar. Paraparesia Espástica Tropical. HTLV.

\section{ABSTRACT}

Low back pain related to Tropical Spastic Paraparesis / Myelopathy associated with HTLV 1 (PET / MAH) becomes relevant because it is a frequent clinical symptom in these patients. Objectives were to assess low back pain and disabilities. METHODS: Fifteen PET / MAH patients were evaluated using three questionnaires: Visual Analog Scale (VAS), Katz Index and Roland-Morris Disability Questionnaire for low back pain. RESULTS: There was a prevalence of low back pain $(93.30 \%)$, and this pain was localized $(85.71 \%)$. The intensity of the pain was moderate $(66.7 \%)$, the majority $(60.0 \%)$ was independent, however $(53.3 \%)$ of them has functional disability associated with low back pain. CONCLUSION: This study showed a high prevalence of low back pain, the higher the level of pain reported by patients, the greater the level of functional limitation presented by them because there is a direct relationship between both.

DESCRIPTORS: Low Back Pain. Paraparesis, Tropical Spastic. HTLV.

\section{RESUMEN}

La lumbalgia relacionada con la paraparesia Espástica tropical / Mielopatia asociada a HTLV 1 (PET / MAH) adquiere relevancia por ser un síntoma clínico frecuente en estos pacientes. Los objetivos fueron evaluar el dolor lumbar y las discapacidades. MÉTODOS: Quince pacientes con PET / MAH fueron evaluados mediante tres cuestionarios: Escala Visual Analógica (EVA), Índice de Katz y Cuestionario de Discapacidad de Roland-Morris para el dolor lumbar. RESULTADOS: Hubo una prevalencia de lumbalgia $(93,30 \%)$, y este dolor fue localizado $(85,71 \%)$. La intensidad del dolor fue moderada $(66,7 \%)$, la mayoría $(60,0 \%)$ fue independiente, sin embargo $(53,3 \%)$ de ellos presenta discapacidad funcional asociada a lumbalgia. CONCLUSIÓN: Este estudio mostró una alta prevalencia de lumbalgia, cuanto mayor es el nivel de dolor reportado por los pacientes, mayor es el nivel de limitación funcional que presentan debido a que existe una relación directa entre ambos.

DESCRIPTORES: Dolor de la Región Lumbar. Paraparesia Espástica Tropical. HTLV.

RECEBIDO EM: 31/01/2021 APROVADO EM: 01/03/2021

\section{Bianca Caroline Silva da Cunha}

Fisioterapeuta, Universidade Federal do Pará, Faculdade de Fisioterapia e Terapia Ocupacional, Pará, PA, Brasil, especialista a atenção à saúde cardiovascular, bacharel em fisioterapia.

ORCID: 0000-0002-8468-6016 


\section{Cristina Maria da Silva}

Fisioterapeuta, Universidade Federal do Pará, Faculdade de Fisioterapia e Terapia Ocupacional, Pará, PA, Brasil, especialista em saúde da Família, bacharel em fisioterapia.

ORCID: 0000-0001-6094-6365

\section{Luzielma Macêdo Glória}

Fisioterapeuta, Universidade Federal do Pará, Faculdade de Fisioterapia e Terapia Ocupacional, Pará, PA, Brasil, mestre em saúde da Amazônia, bacharel em fisioterapia.

ORCID: 0000-0001-5409-4354

\section{Denise da Silva Pinto}

Fisioterapeuta, Universidade Federal do Pará, Faculdade de Fisioterapia e Terapia Ocupacional, Pará, PA, Brasil, PhD em Doenças Tropicais, bacharel em fisioterapia.

ORCID: 0000-0003-4940-8114

\section{George Alberto da Silva Dias}

Fisioterapeuta, Universidade Estadual do Pará, Pará, PA, Brasil, Faculdade de Fisioterapia e Terapia Ocupacional, Pará, PA, Brasil, PhD em Doenças Tropicais, bacharel em fisioterapia.

ORCID: 0000-0002-9807-6518

\section{INTRODUÇÃO}

A Paraparesia Espástica Tropical é uma Mielopatia que está relacionada ao Vírus Linfotrópico de Células T Humanas tipo 1 (HTLV 1). As características clínicas dessa mielopatia são: paraparesia, espasticidade, hiperreflexia de membros inferiores, distúrbios esfincterianos e alterações sensitivas variadas ${ }^{1,2}$. No Brasil, há, aproximadamente, 2,5 milhões de pessoas infectadas pelo vírus HTLV 1 , sendo considerada uma endemia, com média de soroprevalência de $0,45 \%$ entre as pessoas que doam sangue, alcançando $1,8 \%$ na região norte do país ${ }^{3}$.

Um sintoma clínico associado à $\mathrm{Pa}$ raparesia Espástica Tropical/ Mielopatia Associada ao Vírus Linfotrópico de Células $\mathrm{T}$ Humanas tipo 1 (PET/MAH) é a dor lombar, sendo uma queixa recorrente e que faz parte do critério diagnóstico da Organização Mundial da Saúde (OMS) para esta doençą, ${ }^{3,4}$ A freqüência desse sintoma varia de $44 \%$ a $79 \%$ e está relacionada a um maior tempo de evolução da doença, baixo nível funcional e um alto grau de incapacidade. A dor lombar é caracterizada pela localização da dor, que vai da parte inferior do dorso, entre o último arco costal e a prega glútea, com ou sem irradiação para membros inferiores ${ }^{5}$. Nesse contexto, dor é uma sensação sensorial e emocional desagradável e subjetiva, descrita ou decorrente em termos de lesão tecidual ou potencial ${ }^{6}$.

A experiência dolorosa tem características que podem ser contrastantes com múltiplas localizações, distintas descrições e diferentes implicações para aqueles que apresentam incapacidade funcional e alteração da mobilidade ${ }^{7,8}$. Acredita-se que a dor do tipo crônica contribui para as deficiências existentes, exacerbando limitações e diminuindo a mobilidade, conseqüentemente interferindo nas atividades de vida diária ${ }^{8,9}$.

Ainda que a dor seja um sintoma freqüente e expressivo em pacientes com PET/ $\mathrm{MAH}$, que leva à incapacidade e impacto biopsicossocial, há poucos estudos específicos sobre o tema, predominando estudos do tipo relatos de casos como evidência científica ${ }^{10}$. Nesse contexto, o presente estudo torna-se relevante por buscar investigar a dor lombar desencadeada pela PET/MAH, uma vez que esse sintoma clínico é freqüente nesses pacientes, com prevalência que varia de $79 \%$ a $87 \%$. Diante do exposto, o estudo teve como objetivos avaliar a dor lombar e incapacidades relacionadas ao quadro álgico referido pelos portadores de PET/MAH.

\section{MÉTOdOS}

O estudo caracteriza-se por ser quantitativo, descritivo, do tipo transversal, realizado com portadores PET/MAH atendidos no Laboratório de Estudos em
Reabilitação Funcional (LAERF) da Universidade Federal do Pará (UFPA). No período de junho a outubro de 2018.

O presente estudo foi realizado em anuência com os preceitos da Declaração de Helsinque, Código de Nuremberg do Conselho Nacional de Saúde, com aprovação do Comitê de Ética em Pesquisa do Núcleo de Medicina Tropical sob No 063/2011 e aplicação do Termo de Consentimento Livre Esclarecido (TCLE), o qual foi explicado verbalmente e assinado antes da coleta de dados.

Foram incluídos no estudo indivíduos infectados pelo HTLV-1, de ambos os sexos, com idade maior que 18 anos, que não eram co-infectados com outros vírus ou outras situações clínicas que levassem à imunossupressão e que concordaram participar voluntariamente do estudo. Excluídos os portadores de HTLV 1 assintomáticos ou com sintomas não neurológicos.

Depois de aceite na pesquisa, os participantes responderam a três questionários: Escala Analógica Visual (EVA), Índice de Katz e Questionário Roland-Morris de Incapacidades para dor lombar. A EVA possui um escore que varia de grau 0 (nenhuma dor) a 10 (pior dor possível). Em seguida a intensidade é classificada em Leve (graus 0, 1, 2,3), moderada (graus 4, $5,6,7$ ), e intensa (graus 8, 9,10). O Índice de Katz modificado que avaliam seis atividades básicas (banhar-se, vestir-se, ir ao banheiro, transferir-se da cama ou cadeira, 


\section{artigo}

Cunha, B.C.S.; Maria da Silva, C.; Glória, L.M.; Pinto, D.S.; Dias, G.A.S.;

Lombalgia em portadores de paraparesia espástica tropical/mielopatia associada ao HTLV 1

ser continente e alimentar-se), cada função possui escore de 1 a 3 , o qual quanto menor a pontuação mais independente é o paciente e dependendo dessa pontuação

Tabela 1: Tipo de dor, Intensidade, Capacidade funcional e Grau de incapacidade associado à dor lombar em portadores de PET/MAH, Belém/PA, 2018.

$\begin{array}{cccc}\text { VARIÁVEIS } & \begin{array}{c}\text { NÚMERO } \\ \text { ABSOLUTO }\end{array} & \begin{array}{c}\text { FREQÜÊNCIA } \\ \text { RELATIVA (\%) }\end{array} & \text { VARIÁVEIS } \\ \text { Dor lombar } & 12 & 85,71 \% & 0.0162^{*} \\ \text { Localizada } & 02 & 14,29 \% & \\ \text { Irradiada } & & & \\ \text { Intensidade (EVA) } & 2 & 13,3 \% & 0.0283^{*} \\ \text { Leve } & 10 & 66,7 \% & \\ \text { Moderada } & 3 & 20,0 \% & \\ \text { Intensa } & & & \\ \text { İndice de Katz } & 9 & 60,00 \% & 0.6056 \\ \text { Independente } & 6 & 40,00 \% & \\ \text { Semidependente } & & & \\ \text { Q - Roland-Morris de Incapacidade } & 7 & 46,7 \% & 0.0646 \\ \text { Capacitado funcionalmente } & 8 & 53,3 \% & \\ \text { Incapacitado funcionalmente } & & \end{array}$

Gráfico 1: Correlação entre grau de dor e índice de Katz em portadores de PET/ MAH, Belém/PA, 2018.

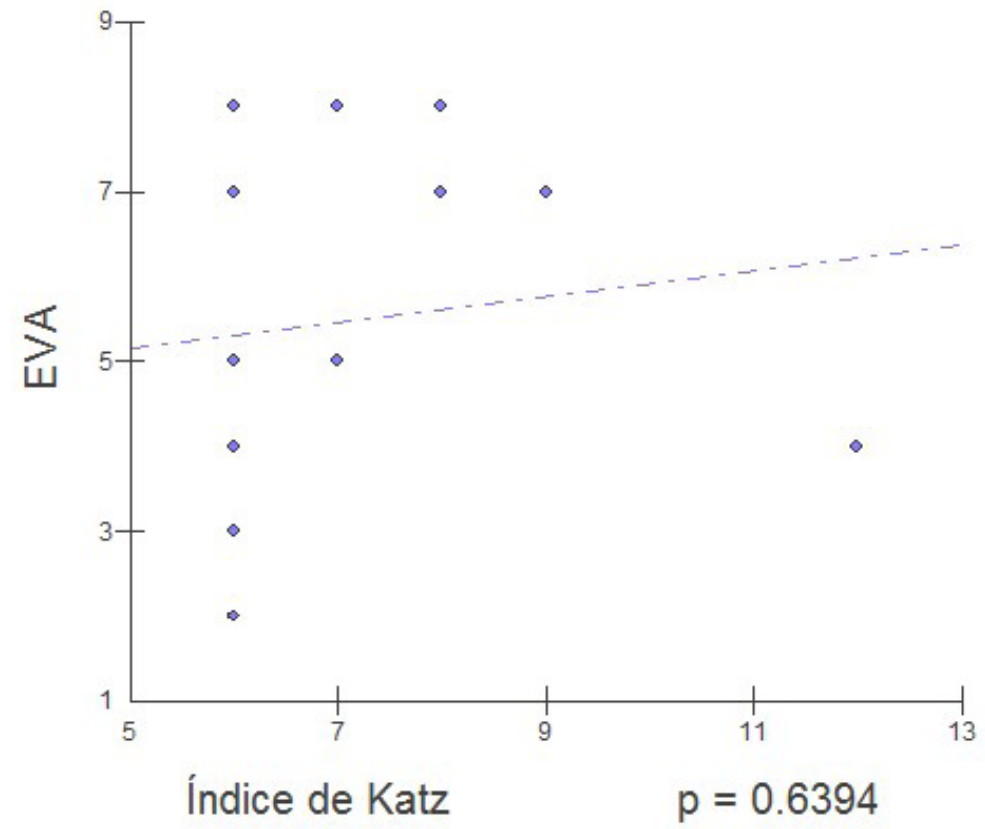

guntas de auto-resposta. As perguntas têm uma resposta dicotômica (sim ou não) e o resultado final corresponde à soma das respostas sim. Este resultado pode variar entre 0 (sem limitação) e 24 (limitação muito grave).

Os dados coletados foram armazenados em um banco utilizando-se o software Microsoft Excel versão 2007, a análise estatística foi realizada no software Bioestat 5.4, onde foram obtidos percentuais das variáveis categóricas e médias das variáveis numéricas. $\mathrm{Na}$ análise inferencial, foram empregados os testes: $G$ de aderência, Qui-quadrado de aderência para proporções esperadas iguais e Correlação linear de Pearson, todos considerando um nível alfa de $5 \%$. Os resultados foram demonstrados a partir de tabelas e gráficos.

\section{RESULTADOS}

Foram entrevistadas 15 pacientes $(\mathrm{n}=15)$ com diagnóstico clínico e molecular de PET/MAH, com idade em média de 55,86 anos, com prevalência maior do gênero feminino $60 \%(\mathrm{n}=09)$. Houve uma prevalência de dor lombar 93,30\% $(n=13)$ em portadores de PET/MAH.

A dor lombar apresentou-se de forma localizada (85,71\%) e com irradiação para membros inferiores $(14,29 \%)$ na amostra estudada, observa-se também a prevalência da intensidade moderada de dor lombar nos portadores de PET/MAH entrevistados, sendo estatisticamente significante ( $p$-valor $=0.0283$ ), além disso, a maioria dos portadores de PET/MAH (60,00\%) era independente, segundo a classificação de capacidade funcional do Índice de Katz. Observa-se que a maioria (53,3\%) dos portadores de PET/MAH tem incapacidade funcional associada à dor lombar, no entanto este resultado não é estatisticamente significante ( $\mathrm{p}$-valor $=0.0646)$ (tabela 1).

No gráfico 1, observa-se a correlação positiva entre os scores da EVA e do Índice de Katz. No gráfico 2, observa-se a correlação positiva entre os scores da EVA e do Questionário de Roland-Morris para Incapacidades geradas pela dor lombar. 


\section{DISCUSSÃO}

Observou-se que a maioria da amostra estudada $(93,3 \%)$ relatou dor lombar. A prevalência de dor lombar em portadores de PET/MAH, descrita na literatura varia de $44 \%$ a $79 \%$, esta variação é explicada em função do tamanho das amostras, sendo que estudos com um número maior tendem a ter maior freqüência, dado este que difere deste estudo, que embora tenha uma amostra pequena, apresentou alta prevalência ${ }^{11}$.

A dor lombar referida pelos portadores de PET/MAH deste estudo pode ser classificada como crônica, pois acompanha o paciente em praticamente toda a evolução da doença, apresentando-se de forma localizada na maioria dos casos $(85,71 \%)$ e em menor freqüência com irradiação para membros inferiores (14,29\%). A OMS propõe como critério diagnóstico para PET/MAH, a presença de lombalgia com irradiação para membros inferiores, e em nosso estudo, essa prevalência foi pequena. Entretanto, esse fato pode ser uma característica específica de

Gráfico 2: Correlação entre grau de dor e Questionário de Roland-Morris em portadores de PET/MAH, Belém/PA, 2018.

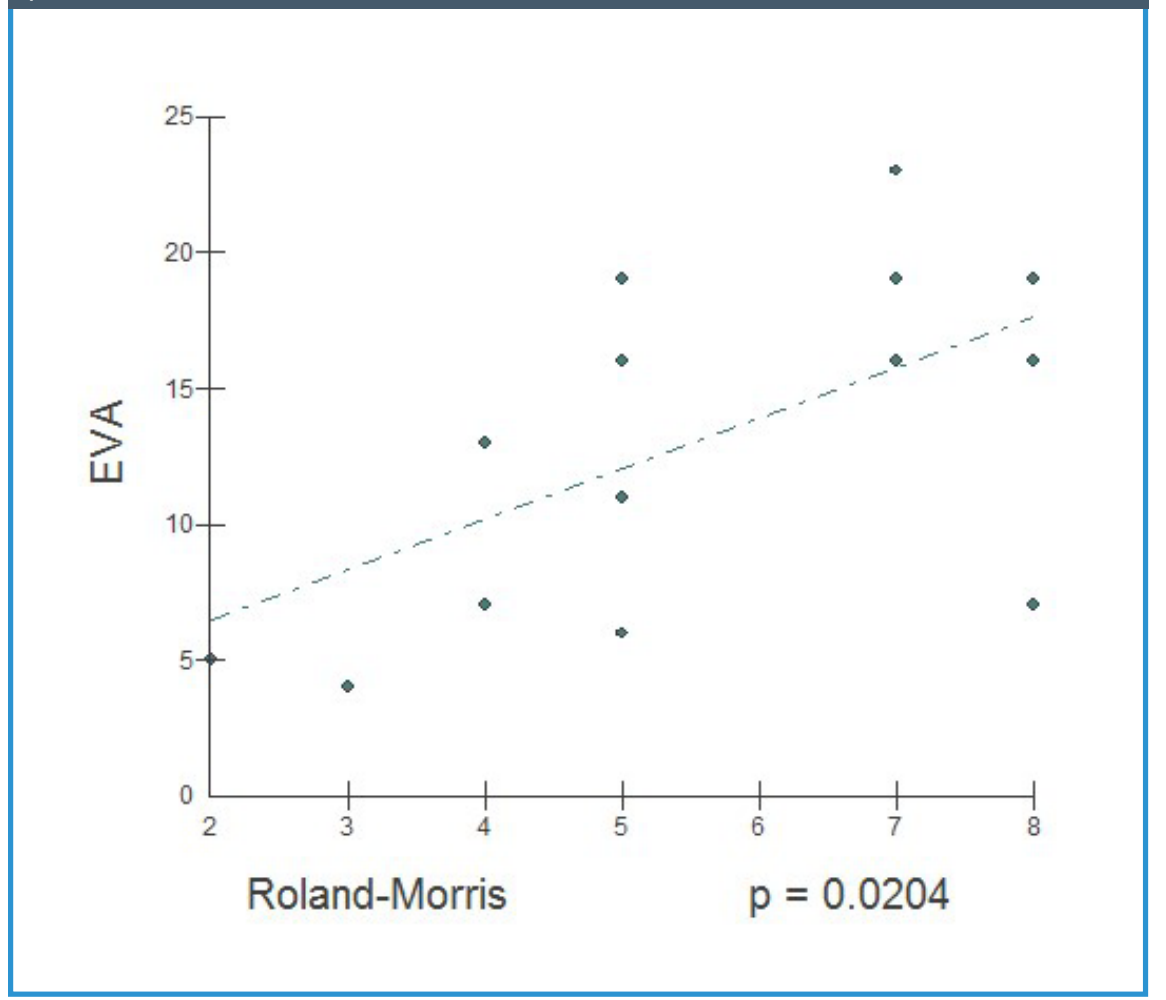

nossa amostra, visto que os pacientes se encontram em tratamento fisioterapêutico há algum tempo, o que pode ter minimizado a presença de irradiações ${ }^{9}$.

$\mathrm{Na}$ maioria dos portadores de PET/ MAH estudados, a intensidade da dor lombar graduada pela EVA foi moderada (66,7\%), apresentando significância estatística ( $\mathrm{p}$-valor $=0,0283$ ). Considerando que era uma dor lombar crônica e que os pacientes apresentavam, em função da doença, uma diminuição da mobilidade, esta intensidade poderia contribuir para maiores restrições, porém um aspecto interessante no presente estudo demonstrou que os sujeitos provavelmente se adaptaram a dor ao longo dos anos, fazendo com que, apesar do incomodo álgico referido, fossem preservadas muitas das atividades gerais de seu cotidiano, mensurados pelo Índice de Katz.

$\mathrm{Na}$ amostra estudada, maioria era independente $(60,00 \%)$, segundo a classificação de capacidade funcional do Índice de Katz. Diante disso, pode-se supor que as deficiências decorrentes da PET/MAH, não influen- ciam na funcionalidade para o desempenho das atividades básicas de vida diária dos indivíduos portadores da doença. No entanto, o estudo ${ }^{12}$ relatou que os sintomas clínicos da PET/MAH interferem no grau de funcionalidade, principalmente as atividades de locomoção (incluindo-se a marcha e subir e descer escadas) e o controle da bexiga. $\mathrm{O}$ estudo $^{13}$ observou uma prevalência de $64 \%$ de quedas e significativo grau de morbidade em portadores de PET/MAH com baixo grau de capacidade funcional. Um histórico elevado de quedas pode resultar em medo, e conseqüentemente, limitar a participação em atividades físicas e sociais, contribuindo ainda mais para o declínio da funcionalidade.

Observou-se que a incapacidade gerada pela dor lombar, avaliada pelo Questionário Roland-Morris, estava presente em mais da metade da amostra $(53,3 \%)$, e isso pode provocar limitações ainda mais importantes. Embora a dor não apareça como fator direto de dependência em muitos estudos, algumas pesquisas epidemiológicas comprovaram a interferência da dor em vários aspectos da vida e relacionaram-se com limitações funcionais ${ }^{14}$. Acredita-se que a ocorrência da dor na região lombar seja predominantemente nociceptiva, de origem espástica, sendo de elevada prevalência ${ }^{15}$. Importante ressaltar o fato de que a incapacidade funcional analisada pelo Questionário de Rolland Morris se relaciona exclusivamente à dor lombar específica desses pacientes, sendo a mesma causada por uma síndrome articular posterior e favorecida pela hipertonicidade da musculatura paravertebral, e não por quaisquer outros mecanismos fisiopatológicos ou aspectos clínicos da doença em si ${ }^{16}$.

Na presente pesquisa, observou-se que não houve correlação positiva estatisticamente significante ( $\mathrm{p}$-valor=0.6394) entre a análise da dor (EVA) e da capacidade funcional (índice de Katz). O estudo ${ }^{17}$ encontrou resultados semelhantes ao verificar que dentre os sintomas sensitivos relacionados à doença, a dor é o mais freqüente e inicial, sendo a dor lombar a mais predominante, porém, não correlacionada positivamente com o grau de capacidade funcional dos indivíduos com PET/MAH.

Por outro lado, houve relação direta entre a dor (EVA) o nível de incapacidade 


\section{artigo}

Cunha, B.C.S.; Maria da Silva, C.; Glória, L.M.; Pinto, D.S.; Dias, G.A.S.

Lombalgia em portadores de paraparesia espástica tropical/mielopatia associada ao HTLV 1

(Questionário Roland-Morris), dessa forma, quanto maior era o nível de dor referido pelos pacientes, maior o nível de limitação apresentados pelos mesmos, e essa associação foi estatisticamente significante ( $\mathrm{p}$-valor $=0$, 0204). O estudo ${ }^{18}$ identificou uma associação significativa entre a incapacidade do indivíduo portador de PET/MAH e a classificação da dor, onde indivíduos com pior incapacidade apresentam maior índice de dor, enquanto os indivíduos menos comprometidos apresentam menor índice de dor.

Por fim, o estudo realizado apresentou como limitação quanto ao tamanho da amostra, que ao se apresentar em número reduzido, permite considerar os resultados apenas para a população em questão. A definição da amostra também pode ser considerada um fator limitante, tendo em vista o fato de ela não ser aleatória.

\section{CONCLUSÃO}

A análise dos dados obtidos através desse estudo permitiu-se concluir que a dor lombar foi o sintoma mais freqüente entre os portadores da PET/MAH, seguida de queixas álgicas em membros inferiores. A dor lombar nos pacientes com PET/MAH caracterizou-se por ser do tipo crônica e em geral localizada, com intensidade moderada.

Os resultados das correlaçóes mostram que o nível de dor lombar tem relação direta com o nível de incapacidade funcional, dessa forma, quanto maior o nível de dor referido pelos pacientes, maior será o nível de limitação funcional apresentado pelos mesmos, para a realização de pequenas atividades do dia-a-dia, no entanto, essa dor não os limita para atividades globais, menos complexas, possivelmente devido à adaptação dos indivíduos a essa dor.

\section{REFERÊNCIAS}

1. Araya N, Sato T, Coller-Reilly A, Yagishita N, Yamano Y. Developing novel treatments for HTLV-1-associated myelopathy (HAM) by investigating molecular pathomechanisms. Jpn J Clin Immun. 2016;39(3):207-12.

2. Barreto FK, Krouri R, Rego FFA, Santos LA, Amarante MFC, Bialuk I, et al. Analyses of HTLV-1 sequences suggest interaction between ORF-I mutations and HAM/TSP outcome. Infect Genet Evol. 2016; 45(1): 420-425.

3. Matsuura E, Nozuma S, TashiroY, Kubota R, Izumo S, Takashima H. HTLV-1 associated myelopathy/tropical spastic paraparesis (HAM/TSP): A comparative study to identify factors that influence disease progression. J neurol Sci.2016; 371(2): 11-116.

4. Dimber R, Guo Q, Bishop C, Adonis A, Buckley A, Kocsis A, et al. Evidence of Brain Inflammation in Patients with $\mathrm{Hu}-$ man T-Lymphotropic Virus Type 1-Associated Myelopathy (HAM): A Pilot, Multimodal Imaging Study Using 11C-PBR28 PET, MR T1-Weighted, and Diffusion-Weighted Imaging. J Nucl Med.2016; 57(12): 1905-1912.

5. Yusuma K, Matsuzaki T, Yamano Y, Takashima H, Matsuoma M, SaitoM. HTLV-1 subgroups associated with the risk of HAM/TSP are related to viral and host gene expression in peripheral blood mononuclear cells, independent of the transactivation functions of the viral factors.J Neurovirol. 2016;22(4):416-30.

6. Alcaíde GG, Ramos JM, Huamaní C, Mendoza C, Soriano V.Human t-lymphotropicvirus1 (htlv-1) andhuman t-lymphotropicvirus 2 (htlv-2): geographical research trendsand collaboration networks (1989-2012). RevlnstMed Trop. 2016; 58(11): 52-45.

7. Cafaia RC, Orsini M, Felicio LR, Sohler MP.Muscular weakness represents the main limiting factor of walk, functional independence and quality of life of myelopathy patients associated to HTLV-1. Arq Neuro Psiquiatr. 2016; 74(4): 109-64.

8. Ciminelli A, Melo F, Copin MC, Proietti AB, Rezende SM. Hematological manifestations of human T lymphotropic virus type 1 infection: a possible association with autoimmune myelofibrosis. Rev Bras HematolHemoter. 2016; 38(1): 78-75.

9. Martin DL, Santos DN, Baptista AF. Pain prevalence, charac- teristics and associated factors in human T-cell lymphotropic virus type 1 infected patients: a systematic review of the literature.Braz J Infect Dis. 2016; 20(6): 598-592.

10. Stratford PW, Riddle DL.A Roland Morris Disability Questionnaire Target Value to Distinguish between Functional and Dysfunctional States in People with Low Back Pain.Physiother Can. 2016; 68(1): 29-35.

11. Proietti ABF, Ribas JGR, Soares BC, Martins ML, Melo GEA, Martins 0 , et al.Infecção e doença pelos vírus linfotrópicos humanos de células T (HTLV-I/II) no Brasil.RevSocBrasMed Trop. 2002; 35 (5): 508-499.

12. Franzoi $A C$, Araújo $A Q$. Disability profile of patients with HTLV-I-associated myelopathy/tropical spastic paraparesis using the Functional Independence Measure (FIM). Spinal Cord.2005; 43(4): 236-40.

13. Facchinetti, LD. Falls in patients with HTLV-I associated myelopathy/tropical spastic paraparesis (HAM/TSP). Spinal 2013; 51(3): 5-222.

14. Ferreira MSM, Ferreira MG. O papel moderador do tipo de família na relação entre incapacidade funcional e qualidade de vida em doentes com lombalgia crônica. CienSaude Colet. 2016; 21 (1): 309-303.

15. Teixeira MJ, Almeida DB, Yeng LT. Conceito de dor neuropática aguda. $O$ papel do nervinervorum na distinção entre dores agudas nociceptiva e neuropática. Rev Dor. 2016; 17 (1): 10-5.

16. Smdja B. Paraplegia associated with HTLV-1 in Martinique. Study of 271 cases including 70 with neuromuscular involvement. Bull SocPatholExot.1993; 86(5): 433-8.

17. Costa CMC, Araújo AQC, Câmara CC, Ferreira AS, Santos TJ, Costa SB, et al. Pain in tropical spastic paraparesis/HTLV-I associated myelopathy patients.Arq Neuro Psiquiatri. 2009; 67(3): 866-70.

18. Netto EC, Brittes C. Characteristics of Chronic Pain and Its Impact on Quality of Life of Patients With HTLV-1-associated Myelopathy/Tropical Spastic Paraparesis (HAM/TSP).Clin J Pain. 2011;27(2):131-5. 